\title{
Aplicación Móvil educativa para facilitar el aprendizaje de la Lengua de Señas del Perú en personas sordomudas
}

\section{Educational mobile application to facilitate the learning of the Sign Language of Perú in deaf and dumb people}

\author{
iD 'Dany Dorian Isuiza Pérez $\quad$ (iD ${ }^{2}$ Leonidas Asto Huamán (iD) ${ }^{3}$ Magaly Roxana Arangüena Yllanes \\ (iD 2Jorge Rafael Diaz Dumont
}

Universidad Nacional Amazónica de Madre de Dios, Perú

Universidad Nacional Autónoma de Tayacaja "Daniel Hernández Morillo". Pampas, Perú

Universidad Nacional de Huancavelica, Perú ${ }^{3}$

Recibido:

Junio 2020

Aceptado:

Setiembre 2020

\section{Resumen}

En el Perú existen más de 532,000 personas sordas con 23 intérpretes y un solo colegio especial donde se enseña el lenguaje de señas, aunque en el país se reconoce oficialmente la lengua de señas peruana (LSP). Esta investigación tuvo como objetivo diseñar una aplicación móvil que facilite el aprendizaje de LSP. Se probó esta aplicación móvil educativa con los estudiantes dentro y fuera del aula, con los padres de familia, y personas del entorno. La aplicación móvil facilitó a las personas con esta discapacidad a mejorar la comunicación versátil, sin necesidad de tener un intérprete a su lado.

Palabras clave: Aplicación móvil, herramienta tecnológica, lenguaje de señas peruana.

\section{Abstract}

In Peru, there are more than 532,000 deaf people with 23 interpreters and a single special school where sign language is taught, although the country is officially recognized as the Peruvian sign language (LSP). This research aimed to design a mobile application that facilitates the learning of LSP. This educational mobile application was tested with students inside and outside the classroom, with parents, and people around them. The mobile application made it easier for people with this disability to improve versatile communication, without the need to have an interpreter by their side.

Keywords: Mobile application, technological tool, Peruvian sign language. 


\section{INTRODUCCIÓN}

La población con discapacidad auditiva, carece de oportunidades laborales y académicas. (Espinosa, Gómez, \& Cañedo, 2012). Debido a que en América Latina, la oferta de herramientas tecnológicas para la población con discapacidad es reducida, y además, costosa por el hecho de ser importadas, hace más difícil poder mejorar su nivel de inclusión social, a través del desarrollo de su potencial en el ámbito laboral y académico, generando una brecha muy acentuada que no permite a las políticas públicas y a los programas de apoyo a la discapacidad, tener significativos avances en esta materia (Alonso \& Díaz, 2008).

A pesar de que las tecnologías de información han crecido a pasos agigantados, los precios se han elevado para adquirir la última herramienta tecnológica que apoye o mejore a esta discapacidad. Este software se construyó debido a que las autoridades regionales, Ministerio de Educación y en especial el Estado Peruano han descuidado a las personas sordomudas. Para Aquino et al., 2012, en su investigación indica que la inclusión educativa de estudiantes con discapacidad representa una prioridad para organismos nacionales e internacionales. En consecuencia, diversas leyes y reglamentos han sido propuestos; no obstante, el liderazgo para caminar a una escuela inclusiva no descansa sólo en las leyes y las autoridades de las instituciones educativas; tampoco se construye necesariamente sobre la base de buenas intenciones.

El éxito de la inclusión educativa depende de los distintos agentes y actores de la sociedad. Comparado con otros tipos de discapacidad, ellos no presentan problemas de aprendizaje, sino más bien dificultades en la comunicación, es preciso resaltar que las personas sordomudas sufren en la inclusión social cuando interactúan con la sociedad, comprar o realizar cualquier tipo de actividades, debido a que no todos los lugares o ámbitos sociales cuentan con intérpretes, para poder establecer un cierto nivel de comunicación.
Se diseñó un software educativo amigable, intuitivo y flexible que permite ejercitar vocabularios básicos a través del uso, entablar una comunicación a través del lenguaje de señas a nivel de los profesionales, niños, padres de familia, etc. Este software tiene niveles de aprendizaje en función al proceso y nivel de aprendizaje de las personas.

Para Asto et al., 2019 en su trabajo de investigación indica que: "los padres de familia o apoderados se sienten mejor motivados con la aplicación, en vista que ahora se pueden comunicar de mejor manera con sus hijos". La investigación anterior es una motivación para desarrollar un aplicativo móvil orientado a todas las personas con bajo recursos económicos y como plataforma a los equipos con pantallas gráficas táctiles, el cual le permite al usuario sordomudo, interactuar con todas las funciones de la aplicación desarrolladas en el software con funciones propias implementadas por parte del dispositivo móvil, para mejorar el proceso de comunicación y enseñanza, de esta manera el nivel de comunicación se incrementa entre las personas sordomudas.

\section{MATERIALES Y MÉTODOS}

Esta investigación es correlacional. La población y muestra fueron 30 personas sordomudas, las cuales se seleccionaron de manera aleatoria; se hicieron equipos de trabajo donde participaron: investigadores, padres de familia e hijos, para poder fortalecer y observar el proceso de aprendizaje de las personas sordomudas.

Asimismo, participó la Dirección Regional de Educación en Madre de Dios Provincia de Tambopata, para poder facilitar y/o entregar la información de las personas que sufren discapacidad auditiva y por ende el habla. Así mismo, no se cuenta con estadísticas del número de personas con esta discapacidad; igualmente, son pocas que acuden a la institución educativa y que necesitan de otro tipo de aprendizaje. 
De igual manera, se trabajó con las personas sordomudas que contaba la institución educativa, incorporando a los padres de familia, con la finalidad de mejorar el proceso de aprendizaje. Se realizaron entrevistas, se aplicaron cuestionarios a la población a los padres de familia y estudiantes, los cuales brindaron buena información, la cual fue recopilada como parte del proceso de análisis y construcción de la aplicación móvil. Estas pruebas se aplicaron a los alumnos de la I.E. Stella Maris de la ciudad de Puerto Maldonado por un periodo de un mes; durante dos semanas se trabajó en aula y las otras dos semanas, con los padres de familia en sus respectivos hogares.

Como parte aplicativa de la investigación se estimuló a los estudiantes y docentes con la aplicación móvil, a través de una capacitación por más de 45 minutos e instalación de la aplicación educativa en los celulares móviles de media gama que tenían los docentes; esta aplicación móvil también funciona en dispositivos de tipo Tablet. Se hizo evidente la inquietud por su uso y luego de 30 minutos se contrastó la capacidad de comunicación entre ellos (estudiantes) y sus docentes; esta prueba duró dos semanas.

Se aplicó la prueba "a de Cronbach" para la validez interna de los instrumentos: la aplicación móvil educativa (p: 0,922), mientras que para el aprendizaje de la Lengua de Señas. ( $p$ : 0,880): para el análisis de los datos se usó el programa SPSS versión 22.

\section{RESULTADOS}

Los resultados obtenidos de esta investigación se muestran en el modelo de variables la aplicación móvil con software educativo y aprendizaje de lenguaje de señas peruana:

\section{Tabla 1}

\section{Distribución de frecuencia de la aplicación móvil educativa}

\begin{tabular}{lcc}
\hline Nivel & n & \% \\
\hline Malo & 1 & 3,3 \\
Regular & 6 & 20,0 \\
Bueno & 15 & 50,0 \\
Muy Bueno & 8 & 26,7 \\
\hline Total & 30 & 100,0 \\
\hline
\end{tabular}

En la Tabla 1, se indica que el $50 \%$ de los encuestados considera que es buena la aplicación móvil, $26,7 \%$ indica que es muy buena.



Figura 1. Interfaz Gráfica de lenguaje de señas peruana: colores primarios y figuras 
En la Figura 1, representa de la interfaz de la aplicación móvil como alternativa a la motivación de esta investigación.

\section{Tabla 2}

Aplicación móvil educativa y aprendizaje de lenguaje de señas peruana, (coeficientesa)

\begin{tabular}{|c|c|c|c|c|c|}
\hline \multirow{2}{*}{ Modelo } & \multicolumn{2}{|c|}{$\begin{array}{l}\text { Coeficientes no } \\
\text { estandarizados }\end{array}$} & \multirow{2}{*}{$\begin{array}{c}\begin{array}{c}\text { Coeficientes } \\
\text { estandarizados }\end{array} \\
\text { Beta }\end{array}$} & \multirow[t]{2}{*}{$t$} & \multirow[t]{2}{*}{$\mathrm{p}$} \\
\hline & B & $\begin{array}{l}\text { Error } \\
\text { estándar }\end{array}$ & & & \\
\hline (Constante) & 1,680 & 1,788 & & 0,939 & 0,356 \\
\hline $\begin{array}{l}\text { Aplicación Móvil } \\
\text { educativa con plataforma } \\
\text { educativa }\end{array}$ & 0,416 & 0,051 & 0,838 & 8,113 & 0,00 \\
\hline
\end{tabular}

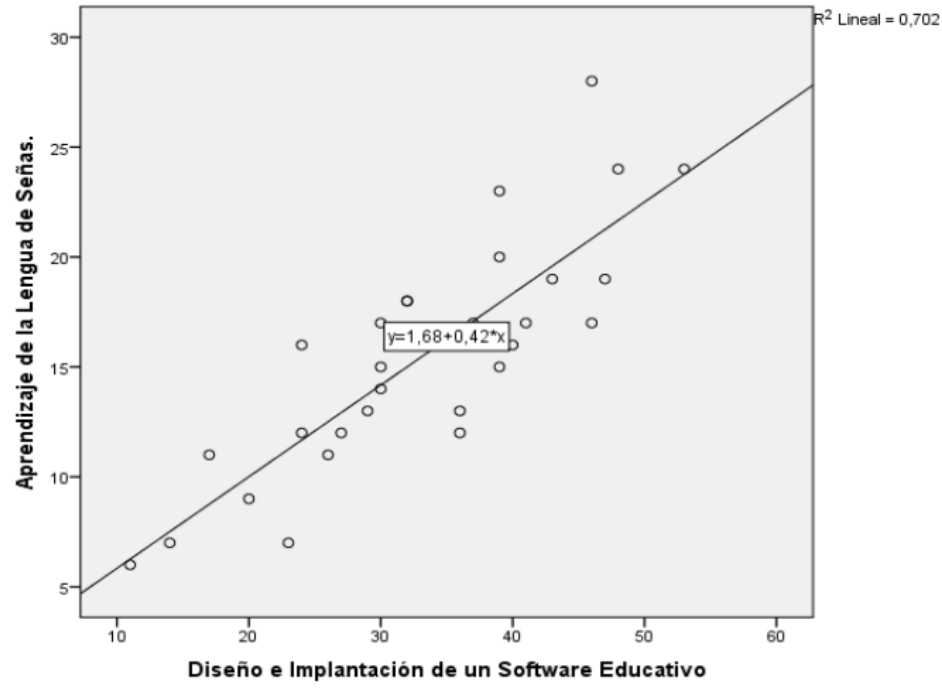

Figura 2. Análisis de regresión lineal entre las variables: aplicación móvil educativa y aprendizaje de lengua de señas peruana.

El gráfico muestra la relación positiva, el ajuste, según el cálculo del R cuadrado, es de 0,702 lo cual indica que es un modelo cuyas estimaciones se ajustan bastante bien a la variable real.

\section{Tabla 3}

Correlaciones de las variables: Aplicación móvil educativa y Aprendizaje del lenguaje de señas peruana.

\begin{tabular}{lccc}
\hline \multicolumn{1}{c}{$\begin{array}{c}\text { Variable } \\
\text { Independiente }\end{array}$} & & Aplicación móvil educativa & $\begin{array}{c}\text { Aprendizaje de } \\
\text { Lengua de Señas. }\end{array}$ \\
\hline $\begin{array}{l}\text { Aplicación móvil } \\
\text { con plataforma }\end{array}$ & $\mathrm{r}$ & 1 & $0,838^{* *}$ \\
educativa & $\mathrm{p}$ & & 0,00 \\
$\begin{array}{l}\text { Aprendizaje de } \\
\text { LSP }\end{array}$ & $\mathrm{r}$ & $0,838^{* *}$ & 1 \\
**. La correlación es significativa & $\mathrm{p}$ & 0,00 & \\
r: correlación de Pearson: $\mathrm{n}(30)$ & & & \\
\end{tabular}


Existe una correlación muy fuerte de 0.838 entre las variables: aplicación móvil con pla- taforma educativa y aprendizaje de lenguaje de señas peruana.

\section{Tabla 4}

Resumen del modelo de las variables: aplicación móvil con plataforma educativa y aprendizaje del lenguaje de señas peruana

\begin{tabular}{|c|c|c|c|c|c|c|c|c|c|}
\hline \multirow[b]{2}{*}{ Modelo } & \multirow[b]{2}{*}{$\mathrm{R}$} & \multirow{2}{*}{$\begin{array}{c}\mathrm{R} \\
\text { cuadrado }\end{array}$} & & \multirow{2}{*}{$\begin{array}{c}\text { Error } \\
\text { estándar } \\
\text { de la } \\
\text { estimación }\end{array}$} & \multicolumn{5}{|c|}{ Estadísticas de cambios } \\
\hline & & & & & $\mathrm{R}^{2}$ & $\mathrm{~F}$ & df1 & df2 & $\mathrm{p}$ \\
\hline 1 & $0,838^{\mathrm{a}}$ & a $\quad 0,702$ & 0,691 & 2,908 & 0,702 & 65,826 & 1 & 28 & 0,00 \\
\hline
\end{tabular}

Donde el coeficiente de variabilidad R cuadrado es de 0.702 como se muestra en la tabla, es un modelo cuyas estimaciones se ajustan bastante bien a la variable real. Los resultados obtenidos dieron a conocer que la aplicación móvil facilita el proceso de aprendizaje en las personas con discapacidad auditiva y de habla.

\section{Tabla 5}

Beneficio de la investigación

\begin{tabular}{lc}
\hline Descripción & $\begin{array}{c}\text { Total, } \\
\text { importe } \$ *\end{array}$ \\
\hline Costo por reclamo & 20.51 \\
Incremento promedio de 5 & $2,458.30$ \\
años de cada reclamo & $166,919.12$ \\
Total & $5,007.57$ \\
Reclamos no deberían & \\
producirse anualmente $(3 \%)$ & \\
\hline${ }^{*}$ Nota $1 \$=3.31 \mathrm{~S} /$ &
\end{tabular}

En ese sentido, se recuperaría la inversión en el sexto año, tal como se aprecia a continuación:

\section{Tabla 6}

\section{Retorno de la inversión}

\begin{tabular}{cccc}
\hline Años & $\begin{array}{c}\text { Beneficios } \\
\text { esperados }\end{array}$ & $\begin{array}{c}\text { Beneficios } \\
\text { esperados } \\
\text { (acumulados) }\end{array}$ & Inversión \\
\hline 1 & $5,007.57$ & $5,007.57$ & $26,223.10$ \\
2 & $5,007.57$ & $10,015.14$ & $26,223.10$ \\
3 & $5,007.57$ & $15,022.71$ & $26,223.10$ \\
4 & $5,007.57$ & $20,030.28$ & $26,223.10$ \\
5 & $5,007.57$ & $25,037.85$ & $26,223.10$ \\
6 & $5,007.57$ & $30,045.42$ & $26,223.10$ \\
\hline
\end{tabular}




\section{DISCUSIÓN}

(Deliyore, 2018) señala "cualquier persona con dificultades de expresión y comprensión del lenguaje oral, ya sea por una ausencia total o parcial de este, pueden utilizar la comunicación alternativa como un medio de acceso al intercambio social". También, (Cano, Muñoz, Collazos, \& Bustos, 2015), menciona que la implementación de la Tecnología de la Información y Comunicación (TIC), ha producido un cambio significativo con la usabilidad de videojuegos que refuerzan el aprendizaje significativo.

Con respecto al lenguaje de señas peruana se hicieron las pruebas y verificación del proceso de ejecución de la aplicación móvil, el software educativo funcionó correctamente con todas las propuestas pedagógicas planteadas por el Ministerio de Educación. No obstante, la institución educativa no es especializada en lenguaje de señas peruana; los docentes se esmeraron para apoyar a la enseñanza aprendizaje de los estudiantes, la interacción que tuvieron los estudiantes y la aplicación móvil no presentó inconvenientes por lo que los niños y los docentes de los diferentes ambientes, mostraron gran interés en usar el aplicativo móvil y a los docentes les ayudó a desarrollar mejor sus sesiones de clases.

Los padres de familia sabían que se les estaba capacitando a sus hijos con el software desarrollado, por lo que también se les enseñó el manejo del aplicativo en sus propios domicilios, al ver el funcionamiento de la aplicación móvil con software educativo se dieron cuenta de la facilidad de comunicación con sus hijos. Otros padres de familia, amigos quisieron tener instalado el aplicativo móvil en sus equipos celulares como en sus tablets, para mejorar el proceso de aprendizaje.

Se utilizó la técnica de observación, cuestionarios, entrevistas a los estudiantes, padres de familia, población y docentes de la institución educativa, para poder recolectar la información y el equipo investigador fortalezca la aplicación móvil con software educativo de acuerdo a las necesidades de los estudiantes, el aplicativo móvil tiene una serie de módulos en las cuales el estudiante o cualquier otra persona podrá aprender a utilizar la lengua de señas peruana.

Los resultados confirmaron las hipótesis, todos los estudiantes que utilizaron el aplicativo móvil con software educativo, demostraron haber fortalecidos el proceso de enseñanza - aprendizaje en comparación con los otros años académicos; así lo ratifican sus docentes, que los niños prestan más atención al aplicativo móvil, que en una explicación normal por parte de sus docentes.

La aplicación móvil desarrollada ha sido aprobada por los estudiantes, padres de familia, docentes, que mencionaron es una aplicación funcional, interfaz gráfica amigable y fácil de usar. La aplicación móvil es una herramienta tecnológica innovadora que apoya a las personas sordomudas a poder socializar con las personas oyentes y habla estándar, que desconocen el lenguaje de señas, quienes se sintieron motivados por tener una aplicación móvil que enseña de forma didáctica basado en el uso de la tecnología. Para Hernández et. al, 2015, en su conclusión menciona que la implementación de la tecnología en un ambiente de aprendizaje es un recurso importante que ayudan a las desventajas existentes con las personas con discapacidad auditiva en un trabajo incansable para la inclusión de las mismas en la sociedad.

Los padres de familia y docentes indicaron que las limitaciones que tienen ellos de adquirir un equipo móvil de gama media o alta, la falta de material accesibles, para poder enseñar a sus hijos el lenguaje de señas peruana, la discriminación de la sociedad hacia las personas sordomudas, limitaciones por parte de sus hijos de utilizar algún tipo de servicio público son limitaciones que no pueden superar y esperan con mucha expectativa que 
en unos años mejore su inclusión en la sociedad.

El casi nulo apoyo por parte del gobierno central a la comunidad sordomuda, durante todo este periodo sólo ha sido publicada una guía de lenguaje de señas peruana, y la ley del lenguaje de señas peruana $y$, aún no se reglamenta hasta la fecha.

Esta aplicación móvil es una primera versión y se deja para trabajos futuros el incremento de más módulos de enseñanza - aprendizaje el cuál debe considerarse en futuras investigaciones.

\section{CONCLUSIONES}

- Esta investigación tiene resultados positivos en el proceso de aprendizaje entre las personas con discapacidad que practican el lenguaje de señas peruana y su contexto de habla natural.

- Es necesario tener tolerancia con las personas sordomudas, debido a que no todos tienen el mismo nivel de discapacidad y brindar apoyo a la persona que lo necesita.

- Se deben desarrollar más aplicativos móviles o software de escritorio, para las personas sordomudas y puedan optar por más de una herramienta de apoyo en la enseñanza-aprendizaje.

- Se deben fortalecer alianzas estratégicas entre las entidades públicas, privadas y ONG's, para que puedan trabajar en las instituciones donde al menos exista una persona sordomuda.

- Se debe de fortalecer el lenguaje de señas peruana en los colegios públicos y privados y enseñar a no discriminar a las personas sordomudas.

\section{REFERENCIAS BIBLIOGRÁFICAS}

Alonso, A., \& Díaz, E. (2008). Universidad y discapacidad: indicadores de buenas prácticas y estándares de actuación para programas y servicios. Esp.Discapacidad Intelectual, 82-98.

Aquino Zúñiga, S. P., García Martínez, V., \& Izquierdo, J. (2012). La inclusión educativa de ciegos y baja visión en el nivel superior: Un estudio de caso. Sinéctica, (39), 01-21.

Asto, L., Ibarra, M., Jimenez, W., Gamboa, J., Limachi, J., Ponce, Y., \& Navarro, A. (2019, October). Application Based on Peruvian Sign Language for the Literacy of Parents of Deaf Children. In 2019 XIV Latin American Conference on Learning Technologies (LACLO) (pp. 278-283). IEEE.

Cano, S., Muñoz, J., Collazos, C., \& Bustos, V. (2015). Aplicación móvil para el aprendizaje de la lectoescritura con FitzGerald para Niños con Discapacidad Auditiva. CBIE-LACLO.

Deliyore, M. (2018). Comunicación alternativa y aumentativa: Acciones y reflexiones para romper el silencio en las aulas. San José.

ElPeruano. (21 de 05 de 2010). Ley que otorga reconocimientooficial a la lengua de señas peruana. www.elperuano.pe.

Espinosa, C., Gómez, V., \& Cañedo, C. (2012). El Acceso y la Retención en la Educación Superior de Estudiantes con Discapacidad en Ecuador. Scielo, 27-38.

Hernández, C., Márquez, H., \& Martínez, F. (2015). Propuesta Tecnológica para el Mejoramiento de la Educación y la Inclusión Social en los Niños Sordos. La Serena.

Perú21, R. (03 de 12 de 2015). En Perú hay 532,000 personas sordas y solo 23 intérpretes. www.peru21.pe.

\section{CORRESPONDENCIA:}

MSc. Leonidas Asto Huamán

leonidasasto@unat.edu.pe 